\title{
Atypical Facial Filler Granuloma: Comparative Histologic Analysis with Paraffinoma
}

\author{
Kang Gyun Park, \\ Eun Sang Dhong, \\ Sik Nam Goong, \\ Jung Kyu Han, \\ Seung Kyu Han, \\ Woo Kyung Kim
}

Department of Plastic and Reconstructive Surgery, Korea University Guro Hospital, Korea University College of Medicine, Seoul, Korea

No potential conflict of interest relevant to this article was reported.

\begin{abstract}
Dermal fillers are generally accepted as safe and well-tolerable cosmetic tools. However, adverse reactions have been reported in the literature. Here, we present a case of atypical facial filler granuloma and compare its histologic features with those of the classic paraffinoma.
\end{abstract}

Keywords: Dermal fillers / Granuloma, foreign-body

\section{INTRODUCTION}

Soft tissue augmentation has become one of the most popular procedures for rejuvenation and aesthetic improvement. This increase in the use of dermal fillers has led to numerous reports on side effects, including foreign body reactions. Foreign body granulomatous reactions following filler injections have different histologic features compared to those following other injectable materials. In this study, we present a case of a granulomatous reaction induced by a filler material with histologic analysis in comparison with paraffinoma, along with a literature review of granulomatous reactions after facial cosmetic filler injections.

\section{CASE REPORT}

We encountered a 58-year-old woman who received a cosmetic

\section{Correspondence: Eun-Sang Dhong}

Department of Plastic and Reconstructive Surgery, Korea University Guro Hospital, Korea University College of Medicine, 148 Gurodong-ro, Guro-gu, Seoul 08308, Korea

E-mail: prsdhong@kumc.or.kr

Received April 5, 2016 / Revised June 7, 2016 / Accepted June 17, 2016 local injection of unknown agents in the nasolabial folds 8 years ago. Two months prior to visiting our clinic, the patient noticed soft-tissue irregularities along the nasolabial fold, which began to increase in size, accompanied by mild local inflammation signs, and migrated into the marionette lines (Fig. 1). Magnetic resonance imaging (MRI) revealed granulomatous tissue occupying the subcutaneous layer in both corners of the mouth (Fig. 2).

Complying with the patient's wishes, the lesions were resected through direct cutaneous approach (Fig. 3). The surgical specimen were sent for histologic analysis, which revealed granulomatous reactions with multinucleated giant cells surrounding multiple foreign materials (Fig. 4). In contrast, histologic findings of paraffinoma, according to the literature review, show giant cells and variable-sized empty vacuoles and cysts (Fig. 5).

\section{DISCUSSION}

Complications associated with filler injection may be immediate, early (within days), or delayed (after weeks to years). Immediate complications include injection site reactions such as erythema, 


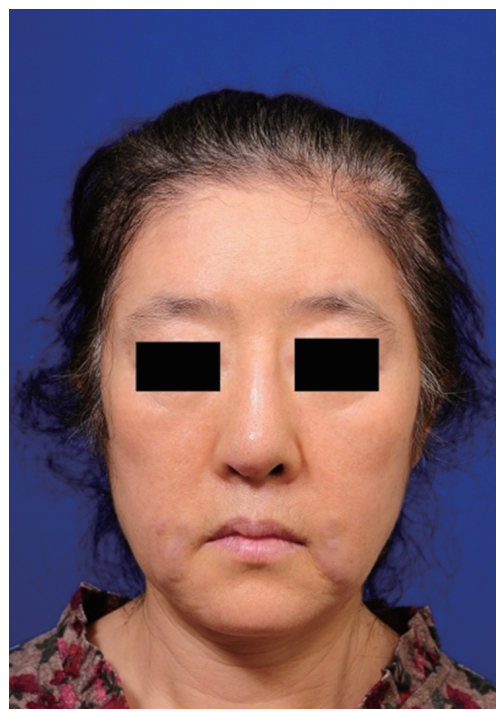

Fig. 1. The patient presented with nodular lesions in both mouth corners after injection of filler materials along the nasolabial folds 8 years ago.
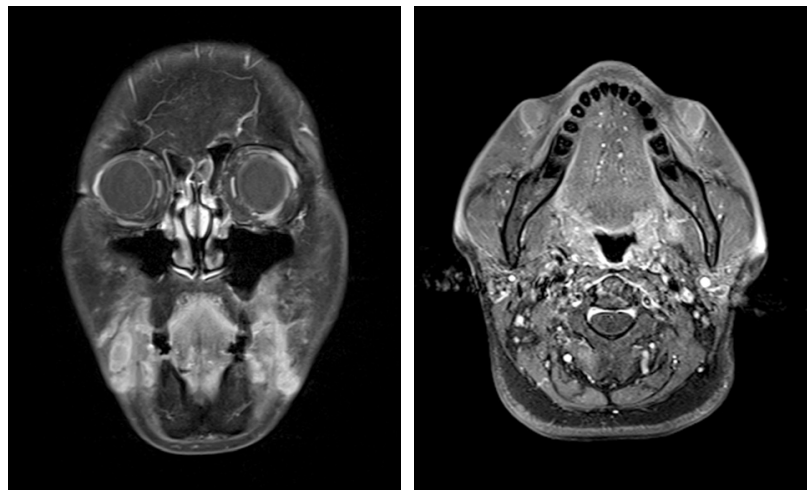

Fig. 2. Magnetic resonance imaging reveals multiple elongated lobulating shape signal change in both corners of the mouth.

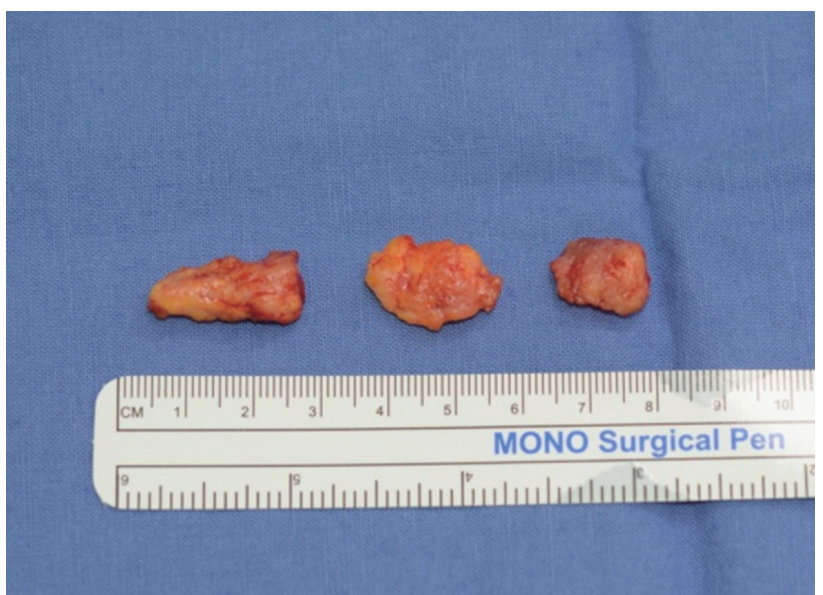

Fig. 3. Resected granulomatous tissues.
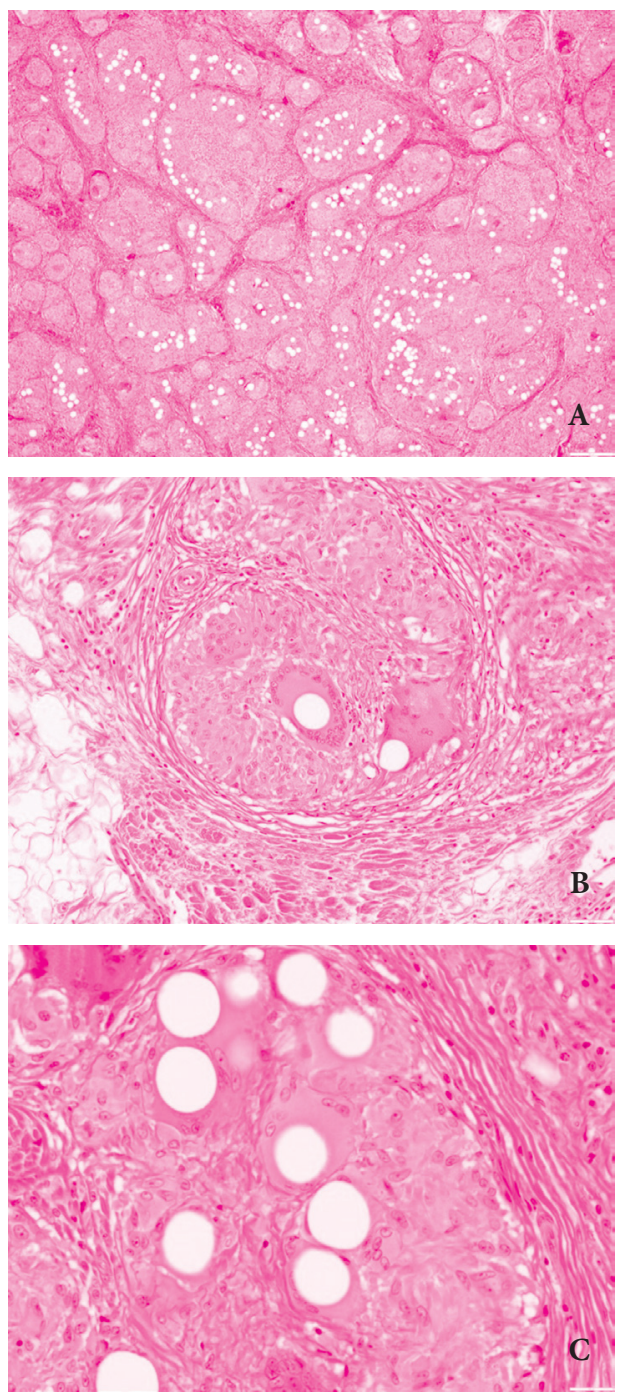

Fig. 4. (A) Histologic examination of the surgical specimen revealed dense lymphohistiocytic infiltrate with multinucleated giant cell. Regular-sized empty vacuoles and cysts $(\mathrm{H} \& \mathrm{E}, \times 40)$. $(\mathrm{B}, \mathrm{C})$ The larger cysts lined by concentric fibrotic stroma, or onion-skin pattern, was also noted $(\mathrm{H} \& \mathrm{E} \times 200)$.

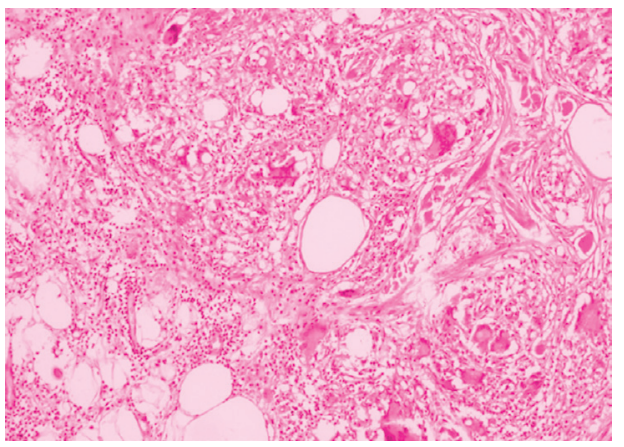

Fig. 5. Paraffin granuloma. A Swiss cheese-pattern of lipogranuloma is observed and consists of giant cells, macrophages, and vacuoles of various sizes $(\mathrm{H} \& \mathrm{E}, \times 100)$. 
edema, pain, and bruising. Early complications include infection (mostly staphylococcal or streptococcal), hypersensitivity reaction, skin discoloration, vascular occlusion, and contour irregularities [1].

Delayed complications include infections (mostly mycobacterial), foreign body granulomatous reaction, migration of implanted material, persistent discoloration, and scarring [2]. Lemperle et al. [3,4] divided foreign body granulomas into three types based on clinical features: cystic granulomas, edematous granulomas, and sclerosing granulomas. Usually, the type of foreign body granulomas primarily depends on the type of filler used, but this is a pattern and not an absolute correlation. Granulomatous foreign body reactions appear after a variable period, between 5 months to 15 years [5].

In our patient, clinical and histological findings suggest the granuloma to be of sclerosing type. Sclerosing granulomas can occur after subdermal implantations of all types of particulate material (e.g., Artecoll, New-Fill/Sculptra, Dermalive, Radiesse) as well as fluid implants (e.g., silicone 350, Aquamid).

Silicone infiltrations have been used in medical practice for years, in both solid and liquid forms, exclusively or in combination with other products. Silicone was initially believed to be innocuous to body tissues, in the same manner as hyaluronic acid, collagen, or polylactic acid. However, evidence of adverse effects of these materials surfaced in the form of foreign body granulomas. In contrast, paraffinoma is characterized by a granulomatous inflammation of the skin due to the introduction of mineral oils of which the paraffin is usually the main component. Paraffin is a mixture of high-molecular-weight hydrocarbons with a colorless-to-white color. Accidental or intentional subcutaneous infiltration has been reported to cause foreign body reactions develop and may even cause ulceration in some cases [6].

Histologically, paraffinoma is a granulomatous foreign body reaction that results from the interstitial application of oily substances [7]. So-called oil cysts with empty centers and rims of foamy or multinucleated macrophages represent the typical histologic finding in paraffinoma.

It is difficult to differentiate paraffinoma from siliconoma in H\&E stain because the Swiss cheese and onion-skin pattern ap- pear in both diseases. Special stainings such as oil red O or Sudan IV stain can demonstrate the presence of oil in the cysts of variable size [8].

Generally, patients with foreign body granulomas are asymptomatic. Common reasons for consultation is the presence of subcutaneous nodules, swelling, and deformation. Many patients are unable to recall exactly the type of filler material injected because of the long delay in granuloma formation and/or the lack of adequate information at the time of injection (infiltrations for many years being performed in clandestine non-healthcare settings).

In the treatment of foreign body granulomas, it is necessary to consider intralesional steroid injection as a primary treatment option. Most foreign body granulomas can be treated successfully with the steroid injections alone. Furthermore, a differential diagnosis should be performed to distinguish granulomas from nodules, and the treatment should be tailored according to the type and cause of granuloma.

Surgical resection have been carried out in extreme cases of granulomas, especially for paraffinomas and siliconomas, although such a treatment is considered highly aggressive and does not guarantee full removal of the widely disseminated filler material, which may be impossible to separate from normal facial tissues [9].

Injecting fillers into the subdermal layer is considered safer than injecting into the intradermal layer in preventing granulomas because the dermis is by far the organ most sensitive and most susceptible to immunologic reactions [3]. Also, it is recommended to not inject large amount of fillers in a single area.

Furthermore, we as physicians should be aware that all filler materials have potential for side effects and that the risk can be diminished but not completely eliminated. Also, we should give a detailed explanation of risks involved to patients beforehand.

\section{REFERENCES}

1. Junkins-Hopkins JM. Filler complications. J Am Acad Dermatol 2010;63:703-5.

2. Sclafani AP, Fagien S. Treatment of injectable soft tissue filler complications. Dermatol Surg 2009;35 Suppl 2:1672-80.

3. Lemperle G, Gauthier-Hazan N, Wolters M, Eisemann-Klein M, 
Zimmermann U, Duffy DM. Foreign body granulomas after all injectable dermal fillers: part 1. Possible causes. Plast Reconstr Surg 2009;123:1842-63.

4. Lemperle G, Gauthier-Hazan N. Foreign body granulomas after all injectable dermal fillers: part 2. Treatment options. Plast Reconstr Surg 2009;123:1864-73.

5. Alijotas-Reig J, Garcia-Gimenez V, Vilardell-Tarres M. Late-onset immune-mediated adverse effects after poly-L-lactic acid injection in non-HIV patients: clinical findings and long-term follow-up. Dermatology 2009;219:303-8.
6. Friedrich RE, Zustin J. Paraffinoma of lips and oral mucosa: case report and brief review of the literature. GMS Interdiscip Plast Reconstr Surg DGPW 2014;3:Doc05.

7. Cohen JL, Keoleian CM, Krull EA. Penile paraffinoma: self-injection with mineral oil. J Am Acad Dermatol 2002;47:S251-3.

8. Uchida Y, Yoshii N, Kubo H, Kanzaki T, Kanekura T. Facial paraffinoma after cosmetic paraffin injection. J Dermatol 2007;34:798-800

9. Lee JM, Kim YJ. Foreign body granulomas after the use of dermal fillers: pathophysiology, clinical appearance, histologic features, and treatment. Arch Plast Surg 2015;42:232-9. 